\title{
Outpatient Latarjet Procedure: Early Complications and Feasibility Validation
}

\author{
Zilber Sebastien $^{1 *}$ and Taieb Raboudi ${ }^{2}$ \\ ${ }^{1}$ Department of Orthopedic and Traumatological Surgery, Henri-Mondor Hospital, France \\ ${ }^{2}$ Department: Henri Mondor Teaching Hospital, Creteil, France
}

*Corresponding author: Zilber Sebastien, Department of Orthopedic and Traumatological Surgery, Henri-Mondor Hospital, 40, avenue de Mal-de-Lattre-de-Assign, 94000 Creteil, France

\begin{abstract}
Background: The Latarjet procedure may be amenable to outpatient elective surgery as it is often performed on young and healthy patients. Thus, ambulatory surgery for Latarjet procedure is recently rapidly increasing in France with very few validation studies. This feasibility study presents early adverse events following outpatient Latarjet surgery compared to Latarjet surgery performed as an inpatient procedure.
\end{abstract}

Hypothesis: There is no difference between outpatient or inpatient Latarjet procedure.

Patients and Methods: Thirty patients operated on an outpatient basis and prospectively followed were compared to 30 patients operated on an inpatient basis. All procedures were performed by the same surgeon. Complication rates as well as clinical outcomes at one year were compared between groups.

Results: Post-operative hematomas which did not require surgery occurred more frequently in outpatient group in which no drain was used. No other differences occurred between groups. All outpatients but one was satisfied with the procedure.

Discusion: The latarjet procedure was found to be safe when performed on an outpatient basis. The addition of wax to the base of the coracoid seemed to diminish hematoma formation.

Level of Evidence: level III

Keywords: Shoulder; Latarjet; Instability; Complications; Ambulatory surgery

\section{Introduction}

Outpatient surgery provides benefits to patients including a decreased exposure to nosocomial infections, a higher rate of satisfaction [1], and a up to a $68 \%$ decrease in direct costs [2]. The Latarjet procedure is now frequently performed on an outpatient basis in up to one third of cases in France in 2017 (Figure 1).

However, the literature regarding the feasibility of outpatient Latarjet surgery is very poor and there is still a need of validation studies [3]. Our aim was to confirm the feasibility of the outpatient Latarjet procedure by comparing the incidence of adverse events and clinical outcomes between patients who underwent inpatient or outpatient Latarjet surgery. Our hypothesis was that the outpatient procedure is both feasible and safe.

\section{Patients and methods}

\section{Patients}

Study inclusion criteria were as follows: patients were considered if they had a diagnosis of recurrent anterior shoulder instability; were deemed candidates for surgical stabilization; had not undergone prior shoulder surgery, and did not have any significant shoulder co-morbidities. All patients underwent primary surgery for anterior shoulder instability using transfer of the coracoid process (Latarjet procedure). All procedures were performed by a Single Surgeon (SZ). Thirty consecutive patients underwent surgery on an outpatient basis between 2013 and 2017 and were prospectively followed. This group was compared to 30 
patients who underwent the Latarjet procedure on an inpatient basis between 2007 and 2012 by the same surgeon.

Surgical protocolA standardized general anaesthesia protocol was followed. An additional interscalene block was administered under ultrasound guidance (single bolus of $20 \mathrm{ml}$ of $0.375 \%$ ropivacaine) associated with $8 \mathrm{mg}$ of a dexamethasone intravenous injection. An open minimally invasive technique was used. The osteotomy of the coracoid process was performed through a deltopectoral approach after the coraco-acromial ligament and pectoralis minor tendon were released and following conjoint tendon exposure and dissection. All harvested coracoid grafts were a minimum of $20 \mathrm{~mm}$ in length. The subscapularis tendon and muscle was split horizontally. Following glenohumeral capsulotomy, bone on the ventral aspect of the coracoid process and on the anteroinferior aspect of the glenoid rim was decorticated. Any remaining anteroinferior bone bankart fragments were resected. The ventral aspect of the coracoid graft was fixed to the inferior portion of the anterior scapular neck such that the transplant was level with the anterior glenoid rim. The coracoid process was drilled with two $3.5 \mathrm{~mm}$ holes and fixed with two 3.5 diameter cortical screws; whilst the glenoid neck was drilled with $2.5 \mathrm{~mm}$ holes to enable compression.

Care was taken to avoid lateral overhang of the graft across the joint line as described by Alain et al. [4]. No additional capsular suture was used. The subscapularis tendon was closed lateral to the graft. Traction on the coracoid graft was avoided all along the procedure to decrease the risk of musculocutaneous nerve injury. The wound was closed in layers with continuous absorbable skin suture. All inpatients had a suction drain inserted. No drain was used in the outpatient group. Sling immobilization was used for one week following surgery. Simple activites of daily living (shower, eating, writing) were immediately permitted. Following one week, self-assisted stretching in all planes was permitted. Running and swimming were allowed after two months, and high-risk sports (rugby, judo...) were allowed after 4 months. All patients were assessed on post-operative day one (by telephone for outpatients); further assessments took place at 1 week, 1 month, 4 months and 12 months post-operative.

\section{Evaluation criteria and statistical analyses}

Readmission rates and early complications were recorded. Shoulder range of motion, recurrent instability, persistent subjective apprehension and shoulder pain were compared between groups at one year. Satisfaction rate with the outpatient protocol was assessed. Continuous variables were compared with the independent t-test and categorical variables with the Fisher exact test; statistical significance was set at 0.05 .

\section{Results}

The 2 groups were comparable at baseline (Table 1). Mean hospital stay in the inpatient group was $2.2 \pm 0.4$ days. One admission for one night occurred in the outpatient group due to dizziness which resolved without further treatment. No complications occurred related to the interscalene block. There were no reoperations, no nerve injuries and no infections in the series.

In the inpatient group, drained blood volume prior to drain removal was negligible in 18 patients, less than $100 \mathrm{cc}$ in 11 patients and $>100 \mathrm{cc}$ in one patient. All drains were removed on post-operative day one. Seven hematomas occurred within the first 3 weeks following surgery: two in the inpatient group and five in the outpatient group (Figure 2). Four of these hematomas, two in each group, discharged and healed spontaneously. All others healed spontaneously without fistulization. One of these hematomas occurred in a patient with the Factor V Leiden defect (outpatient group). Three of these hematomas, with fistulization twice, occurred in the first 9 patients in the outpatient group and induced a change in the surgical technique. In the following 21 patients, prior to closure, the osteotomy of the coracoid feet was explored, washed and waxed prior to closure during which time a blood clot was typically found. Wax was never used for patients in the inpatient group.

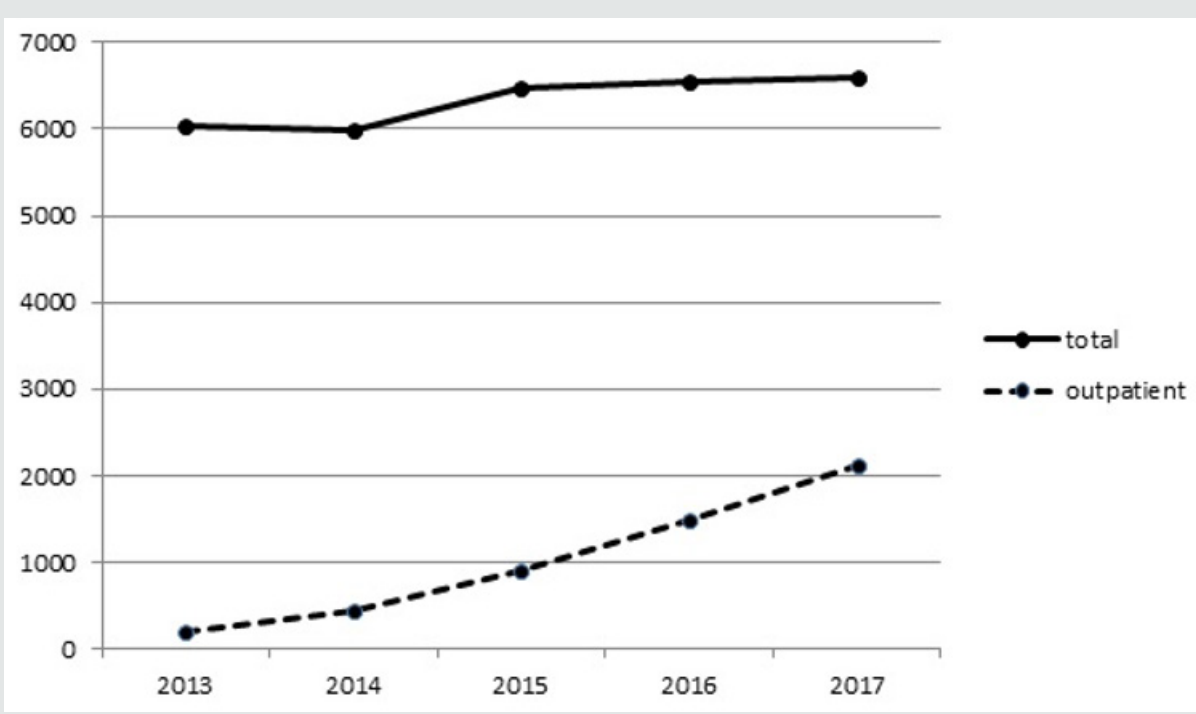

Figure 1: Latarjet procedures statistics in France between 2013 and 2017 (ATIH, technical agency of information on hospitalization, www.atih.sante.fr). 


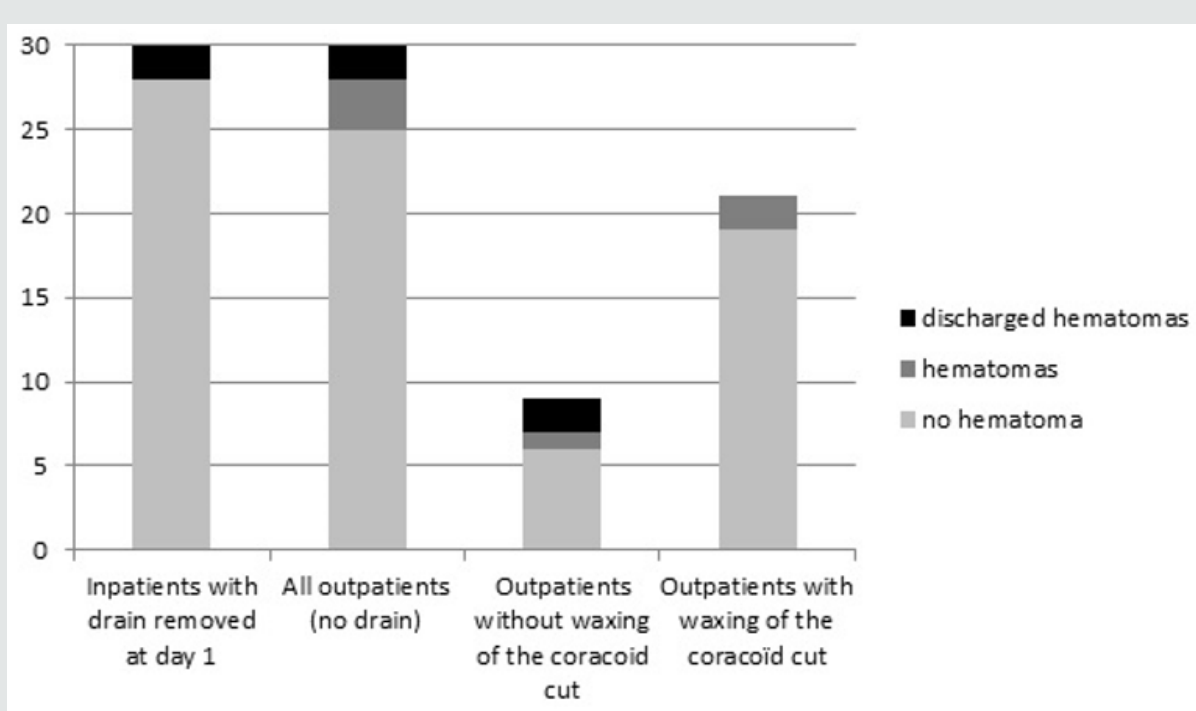

Figure 2: Hematomas formation by group. There was more hematomas in the outpatient group ( $\mathrm{p}<0.01)$, all hematomas healed spontaneously.

All outpatients but one were satisfied with the procedure. One patient indicated that he would have preferred a one night hospital stay due to postoperative discomfort. At final 12 month followup, no patient had experienced further instability. Six patients

answered positively for subjective persistent apprehension in both group (20\%). One third of patients of each group have reported occasional shoulder pain. Loss of external rotation was found in half of patients of each group (Table 1).

Table 1: Demographic variables and clinical results at final follow-up Laxity was defined as passive external rotation $>80$ degrees; $\mathrm{M}=$ male, $\mathrm{F}=$ female.

\begin{tabular}{|c|c|c|c|}
\hline Variables & Inpatients $(n=30)$ & Outpatients $(n=30)$ & $\mathbf{p}$ \\
\hline \multicolumn{4}{|c|}{ Pre-operative status } \\
\hline Age (years), (mean \pm SD) & $25.47 \pm 7.77$ & $28.23 \pm 9.27$ & NS \\
\hline Gender & $3 \mathrm{~F} / 27 \mathrm{M}$ & $6 \mathrm{~F} / 24 \mathrm{M}$ & NS \\
\hline Body mass index (mean \pm SD) & $22.83 \pm 2.11$ & $24.89 \pm 3.74$ & NS \\
\hline Patients operated on the dominant side & 15 & 11 & NS \\
\hline Patients with laxity & 0 & 1 & NS \\
\hline \multirow{3}{*}{ Level of sports } & Competition : 10 & Competition : 7 & NS \\
\hline & Recreational : 8 & Recreational : 18 & \\
\hline & None : 12 & None : 5 & \\
\hline \multirow{5}{*}{ Cause of injury } & Sport : 16 & Sport : 21 & NS \\
\hline & Domestic : 7 & Domestic : 7 & \\
\hline & Traffic accident : 4 & Traffic accident : 1 & \\
\hline & Assault : 2 & Assault : 1 & \\
\hline & Seizure : 1 & & \\
\hline Number of dislocation before surgery & $7.9 \pm 7.18$ & $7.47 \pm 5.99$ & NS \\
\hline $\begin{array}{l}\text { Time from injury to surgery (years) } \\
\text { (mean } \pm S D)\end{array}$ & $3.87 \pm 5.27$ & $6.12 \pm 9.97$ & NS \\
\hline \multicolumn{4}{|c|}{1 year follow-up status } \\
\hline Redislocation & 0 & 0 & - \\
\hline $\begin{array}{l}\text { Patients with persistent subjective } \\
\text { apprehension }\end{array}$ & $6(20 \%)$ & $6(20 \%)$ & NS \\
\hline Patients with persistent pain & $11(37 \%)$ & $10(33 \%)$ & NS \\
\hline Patients with loss of external rotation & $17(57 \%)$ & $15(50 \%)$ & NS \\
\hline
\end{tabular}




\section{Discussion}

This study confirms the safety of the Latarjet procedure performed on an outpatient basis. Almost all patients were satisfied with the procedure. There was no difference at one year between patients who underwent surgery on an outpatient or inpatient basis. Besides, the clinical results of both groups according to persistent pain and apprehension and loss of external rotation were similar to what was already reported in the literature [5-9]. The use of interscalene block with long-acting local anesthetic is a well-accepted approach that produces 8 to 12 hours of postoperative analgesia [10]. Intravenous dexamethasone typically results in prolonged block duration to 18 hours providing a very good acceptance of the outpatient procedure [11]. However, the interscalene block may lead to respiratory complications and the supraclavicular block has been described as a safe alternative technique $[12,13]$.

Given the high incidence of post-operative hematomas seen early in the study, this complication was of significant concern. Three hematomas occurred in the first 9 patients in the outpatient protocol. Two of them fistulized 3 weeks after the surgery; all healed spontaneously without further treatment. Complications of the Latarjet procedure were evaluated in a recent meta-analysis which demonstrated that hematomas required draining with an incidence of $0.5 \%$ [14]. Metais et al. have reported 2 hematomas which require surgical irrigation upon 104 open Latarjet [15]. The incidence of hematoma formation not requiring drainage was not reported in these studies. Frank et al. have reported 1 case of hematoma and 1 case of fistulization which did not require surgery upon 133 cases [16]. None of the hematomas of our study did require surgery. We think that simple hematomas may be underreported in the literature. It is possible that the difference in the incidence of hematoma formation between our two groups was related to the retrospective nature of the review process for the inpatient group which may have led to underreporting as well.

Three hematomas had occurred in the first nine patients in the outpatient group. These hematomas were thought to occur from the osteotomized coracoid feet. Given the lack of drain use in the outpatient group, we sought an alternative solution by way of application of Horsley wax on the coracoid osteotomy feet instead of using a short-term drain as reported by Bohu et al. [3]. Only two hematomas, which did not fistulized, occurred in the following 21 outpatients in whom wax was used. It should be noted however that the use of Horsley wax has been reported to lead to foreign body granulomas in rare instances $[17,18]$. Its use should be noted in the operatory report. The study has certain strengths including similar demographic and surgical technique between groups at baseline, but some limitations should be acknowledged. The retrospective analysis for the inpatient group may have led to underreporting of simple hematomas. Patients were moreover not randomly enrolled and the study may be prone to biases inherent in this design. Patients did not have CT-scan so that mal-union and non-union could not have been analyzed even if the study focused on early adverse events.

\section{Conclusion}

The open minimally invasive Latarjet procedure for shoulder stabilization is increasingly performed on an outpatient basis. We did not observe any differences at one year follow-up between outpatient and inpatient group. However, a high incidence of hematoma formation was observed early in the study, which led to a minimal change in surgical technic to fit the ambulatory protocol. No other differences in complications were noted between groups.

\section{References}

1. Zoutman D, Pearce P, McKenzie M, Taylor G (1990) Surgical wound infections occurring in day surgery patients. Am J Infect Control 18(4): 277-282.

2. Heath P, Ogg T, Hall C, Brownlie G (1990) The cost of day surgery. Health Trends 22: 109-111.

3. Bohu Y, Klouche S, Gerometta A, Herman S, Lefevre N (2016) Outpatient Latarjet surgery for gleno-humeral instability: Prospective comparative assessment of feasibility and safety. Orthop Traumatol Surg Res 102(4): 507-512.

4. Allain J, Goutallier D, Glorion C (1998) Long-term results of the Latarjet procedure for the treatment of anterior instability of the shoulder. J Bone Joint Surg Am 80(6): 841-852.

5. Baverel L, Colle PE, Saffarini M, Anthony Odri G, Barth J (2018) Open Latarjet Procedures Produce Better Outcomes in Competitive Athletes Compared With Recreational Athletes: A Clinical Comparative Study of 106 Athletes Aged Under 30 Years. Am J Sports Med 46(6): 1408-1415.

6. Neyton L, Young A, Dawidziak B, Visona E, Hager JP, et al. (2012) Surgical treatment of anterior instability in rugby union players: clinical and radiographic results of the Latarjet-Patte procedure with minimum 5-year follow-up. J Shoulder Elbow Surg 21(12): 1721-1727.

7. Bouju Y, Gadéa F, Stanovici J, Moubarak H, Favard L (2014) Shoulder stabilization by modified Latarjet-Patte procedure: results at a minimum 10 years' follow-up, and role in the prevention of osteoarthritis. Orthop Traumatol Surg Res 100(4): 213-218.

8. Hurley ET, Lim Fat D, Farrington SK, Mullett H (2019) Open Versus Arthroscopic Latarjet Procedure for Anterior Shoulder Instability: A Systematic Review and Meta-analysis. Am J Sports Med 47(5): 12481253.

9. Bah A, Lateur GM, Kouevidjin BT, Bassinga JYS (2018) Chronic anterior shoulder instability with significant Hill-Sachs lesion: Arthroscopic Bankart with remplissage versus open Latarjet procedure. Orthop Traumatol Surg Res 104(1): 17-22.

10. Conroy BP, Gray BC, Fischer RB, Del Campo LJ, Kenter K (2003) Interscalene block for elective shoulder surgery. Orthopedics 26(5): 501-503.

11. Chalifoux F, Colin F, StPierre P, Godin N, Brulotte V (2017) Low dose intravenous dexamethasone ( $4 \mathrm{mg}$ and $10 \mathrm{mg}$ ) significantly prolongs the analgesic duration of single-shot interscalene block after arthroscopic shoulder surgery: a prospective randomized placebo-controlled study. Can J Anaesth 64(3): 280-289.

12. Guo CW, Ma JX, Ma XL, Lu B, Wang Y, et al. (2017) Supraclavicular block versus interscalene brachial plexus block for shoulder surgery: A metaanalysis of clinical control trials. Int J Surg 45: 85-91.

13. Hussain N, Goldar G, Ragina N, Banfield L, Laffey JG, et al. (2017) Suprascapular and Interscalene Nerve Block for Shoulder Surgery: A Systematic Review and Meta-analysis. Anesthesiology 127(6): 9981013.

14. Griesser MJ, Harris JD, McCoy BW, Hussain WM, Jones MH, et al. (2013) Complications and re-operations after Bristow-Latarjet shoulder 
stabilization: a systematic review. J Shoulder Elbow Surg 22(2): 286292.

15. Metais P, Clavert P, Barth J, Boileau P, Brzoska R, et al. (2016) Preliminary clinical outcomes of Latarjet-Patte coracoid transfer by arthroscopy vs. open surgery: Prospective multicentre study of 390 cases. Orthop Traumatol Surg Res 102(8): 271-276.

16. Frank RM, Gregory B, OBrien M, Bernardoni E, et al. (2019) Ninety-day complications following the Latarjet procedure. J Shoulder Elbow Surg 28(1): 88-94
17. Faghahati S, Gleizal A, Beziat JL (2013) Inflammatory granuloma of iliac bone harvest site: a rare complication of Horsley bone wax. Ann Chir Plast Esthet 58(4): 359-361.

18. Das JM (2018) Bone Wax in Neurosurgery: A Review. World Neurosur 116: $72-76$

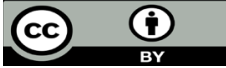

This work is licensed under Creative Commons Attribution 4.0 License

To Submit Your Article Click Here: Submit Article

DOI: $10.32474 /$ OSMOAJ.2019.03.000156

$\begin{gathered}\text { Orthopedics and Sports Medicine } \\ \text { Open Access Journal }\end{gathered}$
Assets of Publishing with us
- Global archiving of articles
- Immediate, unrestricted online access
- Rigorous Peer Review Process
- Authors Retain Copyrights
- Unique DoI for all articles

